\title{
Global Intensity Correction in Dynamic Scenes
}

\author{
P.J. Withagen · K. Schutte • F.C.A. Groen
}

Received: 7 November 2005 / Accepted: 22 April 2009 / Published online: 1 July 2009

(C) The Author(s) 2009. This article is published with open access at Springerlink.com

\begin{abstract}
Changing image intensities causes problems for many computer vision applications operating in unconstrained environments. We propose generally applicable algorithms to correct for global differences in intensity between images recorded with a static or slowly moving camera, regardless of the cause of intensity variation. The proposed intensity correction is based on intensityquotient estimation. Various intensity estimation methods are compared. Usability is evaluated with background classification as example application. For this application we introduced the PIPE error measure evaluating performance and robustness to parameter setting. Our approach retains local intensity information, is always operational and can cope with fast changes in intensity. We show that for intensity estimation, robustness to outliers is essential for dynamic scenes. For image sequences with changing intensity, the best performing algorithm (MofQ) improves foregroundbackground classification results up to a factor two to four on real data.
\end{abstract}

P.J. Withagen was during this research associated with Electro Optics Group, TNO Defence, Security and Safety, and Informatics Institute, Faculty of Science, University of Amsterdam

P.J. Withagen $(\bowtie)$

$\mathrm{X}$-Ray Imaging Innovation, Philips Healthcare, Veenpluis 6, P.O. Box 10.000, 5680 DA Best, The Netherlands

e-mail: Paul.Withagen@philips.com

\section{K. Schutte}

Electro Optics Group, TNO Defence, Security and Safety, P.O. Box 96864, 2509 JG The Hague, The Netherlands

e-mail: Klamer.Schutte@tno.nl

F.C.A. Groen

Informatics Institute, Faculty of Science, University of Amsterdam, Kruislaan 403, 1098 SJ Amsterdam, The Netherlands e-mail: Groen@science.uva.nl
Keywords Intensity correction - Intensity variation . Camera calibration · Pixel classification · Time-varying imagery

\section{Introduction}

Computer vision has proved very successful in well-constrained industrial environments (for instance when illumination, object types, and orientations are known). However, in many practical applications, including airborne or remote sensing, medical imaging, face recognition, outdoor robotics, and surveillance applications, the environment can hardly be controlled. Illumination changes by lights switching on or off, or by clouds moving in front of the sun. Automatic gain control (AGC), white balance and iris are often applied to optimally map the amount of reflected light to the digitizer dynamic range. However, when scene content changes they cause changing image intensity over time.

Problems then arise with many algorithms that assume Constant Image Brightness (CIB) or that are based on the Brightness Constancy Constraint Equation (BCCE). Applications where image intensity changes cause problems include background subtraction, object tracking, video coding image retrieval, stereo matching and optical flow computation. Of course, applications will benefit only when not already using (local) normalization.

In this paper we introduce algorithms for correction of global intensity changes in image sequences. The algorithms estimate and correct for global temporal intensity variations. They can be applied as pre-processing step for other image processing algorithms as mentioned above. We limit ourselves to closed form solutions to guarantee possible implementation for real-time applications. Based on a model of 
a CCD camera a number of algorithms are proposed. The algorithms are evaluated on both simulated and real images.

Evaluation of the algorithms is performed on three criteria. First, the accuracy of the parameter estimation is evaluated using the sum of squared differences between a reference image and the corrected image. Second, the usability of the correction is evaluated using the performance of a representative post processing algorithm. Third, the robustness of the post processing is evaluated. For all evaluation methods we will show the impact of statistical outlier removal.

For real-world application we will focus on the foreground-background classification problem. We use the popular online Expectation Maximization (EM) algorithm to estimate a multi-Gaussian model of the background color for each pixel, see Priebe (1994), Stauffer and Grimson (2000), Withagen et al. (2004), Withagen (2006). The model is used to classify pixels as foreground or background. This is an essential step in many surveillance applications.

This paper is structured as follows: in Sect. 2 we discuss existing techniques to handle changes in intensity. We will introduce our model of changing intensity in Sect. 3 . There we will consider the differences between changes in intensity caused by the combination of a changing scene and automatic gain control, and by changing illumination. In Sect. 4 the proposed methods are described, according to a model of the CCD camera given in Appendix A. These methods are evaluated by both simulation and experiments on real images in Sect. 5. Finally, conclusions are presented in Sect. 6.

\section{Previous Work}

There exists an extensive amount of literature concerning applications like moving object detection, stereo matching or optic flow calculation. Considering that these algorithms normally expect constant image intensity, it is surprising how little work is done in the area of intensity correction. In this section we shortly introduce the different techniques that are available for intensity correction. We evaluate them on accuracy, usability and computational complexity.

The intended algorithm will be used for dynamic scenes with moving objects. These objects cause a scene change and can decrease the accuracy of the intensity correction. It is therefore important to be robust against outliers, as will be shown in the experiments in Sect. 5. The simplest way of dealing with changes in intensity is ignoring all intensity information. Intensity invariants (Siebert 2001) or normalized colors can be used. Instead of intensity information other features can be used, for example color (Greiffenhagen et al. 2001; Horprasert et al. 1999), edges (Jabri et al. 2000; Javed and Shah 2002), or depth (Harville et al. 2001). However, using these features disregards the information available in the intensity.
Local changes in intensity are beyond the scope of this paper. However, techniques dealing with local changes could be used to correct for global changes in intensity. For example, the pixel ordering in a neighborhood (Xie et al. 2004) can be used, one can use a maximum likelihood estimate in an image region (Ohta 2001), or one can use the low frequencies (Toth et al. 2000). For background modelling, one can consider shadow detection, see for example Withagen (2006), Withagen et al. (2008), Horprasert et al. (1999), Hsieh et al. (2003), Pavlidis and Morellas (2001). The use of Kalman prediction to the kernels in the EM model (Stauffer and Grimson 2000) is another example of a local technique in conjunction with background modelling. A drawback of these techniques is that they ignore the fact that all pixels change simultaneously. This leads to a less accurate estimate of the global effect.

Literature reports complicated methods: dynamic histogram warping changes image intensities such that the histograms of the two images become equal (Cox et al. 1995), by an iteratively weighted least squares estimation, the bias, gain and gamma of an image can be estimated and corrected for Tsin et al. (2001), and estimation of the gain and bias together with optic flow allows the use of optic flow under global intensity changes (Altunbasak et al. 2003). A drawback of these methods is the high computational complexity. This makes real-time implementation difficult and expensive. Also, Cox et al. (1995) and Altunbasak et al. (2003) do not perform outlier removal.

Considering background modelling techniques, a frequently used approach for dealing with changes in image intensity is relying on the adaptation speed of the background modelling technique (McKenna et al. 2000). However, this adaptation will only resolve the problem for relatively slow changes in intensity, and it is difficult to tune the update speed of the model. A high update speed may learn slow objects into the background model (missed objects), while a low update speed can be unable to adapt the model fast enough to cope with changes in intensity (false alarms), see Toyama et al. (1999). No single update speed guarantees acceptable results for all possible situations.

An approach, closely related to relying on the adaptation of the background classification algorithm, is setting a limit on the fraction of allowed foreground pixels (e.g. $70 \%$ ). When this fraction is exceeded another background model (if available) is chosen or the background model is re-initialized (Javed and Shah 2002; Toyama et al. 1999). The performance will decrease in applications where illumination or gain changes frequently occur, because after reinitialization a new background model must be learned. During each learning period classification results are unreliable.

The idea of using multiple instances of a scene taken under different illumination conditions is also considered in Hager and Belhumeur (1998), Hischof et al. (2004). They 
use an eigenspace method to overcome complex changes in illumination (not only intensity but also illumination direction may change). An interesting approach in conjunction with image retrieval is given in Jacobs et al. (1998). Their method assumes that the pixel-wise image ratio of two images from the same object is simpler than the ratio of two different objects (they define simplicity as based on the complexity of the algebraic function needed to locally approximate the shape of the image). This assumption allows for object comparison under complex changes in illumination. Only one measure of similarity is calculated for the entire image. Therefore, usability is restricted to applications like face recognition and image retrieval, so this is less general than the scope of this paper, were we are aiming at a generally applicable method.

A useful approach for real-time applications is the direct calculation of the intensity difference between two images. This is done using the average (He et al. 2003) or a least squares estimate (Kamikura et al. 1998). However, these methods are sensitive to outliers.

In this paper we want to develop a method for the correction of global changes in intensity for a static or slowly moving camera that overcomes the above limitations. The method should be generally applicable and insensitive to outliers in dynamic scenes. It should make optimal use of local intensity information. Furthermore, it should have low computational cost so that it can be implemented in realtime.

\section{Model Description}

In this section we present a correction algorithm for global changes in intensity. We then introduce a simplified model of a CCD camera. This model has been experimentally verified for a range of cameras in Withagen et al. (2007).

\subsection{Global Intensity Correction}

The goal of this paper is to correct for global differences in intensity between two images. Consider two images $i_{r}$ and $i_{t}$ depicting an equal scene at different time instances. There is global difference in intensity between the two images. We intend to correct for this intensity difference by

$i_{t, \text { corrected }}=\frac{i_{t}}{a}$

with $a$ the apparent gain factor. $i_{t, \text { corrected }}$ and $i_{r}$ have equal global intensity. We will give an equation for $a$ in this paper.

\subsection{Model of CCD Cameras}

Based on a general model ${ }^{1}$ of CCD cameras (Healey and Kondepudy 1994), we report in Appendix A a simplified CCD model with added gamma correction. This model is based on experimental evaluation described in Withagen et al. (2007, 2005). An important conclusion from this work is that it has been shown that: "It is wrong to pick a general model and assume its validity. It is important to validate the model for the specific camera used."

For the cameras that adhere to the model, the experimental results allow for simplification of the model. For sufficiently large intensity values, both offset and additive noise can be neglected. The simplified model of the CCD camera to be used in the remainder of this paper is now given by

$i_{t}=g_{t}^{\gamma}\left(h_{t} i_{0}+N_{\mathrm{S}}\right)^{\gamma}$,

with $g_{t}$ the camera gain, $i_{0}$ the scene irradiance, $h_{t}$ a factor related to the camera shutter time, iris size and scene illumination, $N_{\mathrm{S}}$ signal dependent noise and $\gamma$ the value of the gamma function.

\subsection{The Naive Gain Factor}

The image recorded at time $t$ will be compared to some reference image $r$ recorded earlier. These images are given by

$i_{t}=g_{t}^{\gamma}\left(h_{t} i_{0}+N_{\mathrm{S}}\right)^{\gamma}$

$i_{r}=g_{r}^{\gamma}\left(h_{r} i_{0}+N_{\mathrm{S}}\right)^{\gamma}$.

Changes in image intensity can be caused by either changes in the camera gain $g_{t}$ or changes in the illumination intensity, iris or shutter resulting in a changed $h_{t}$.

From (1) follows that

$a=\frac{g_{t}^{\gamma}\left(h_{t} i_{0}+N_{\mathrm{S}}\right)^{\gamma}}{g_{r}^{\gamma}\left(h_{r} i_{0}+N_{\mathrm{S}}\right)^{\gamma}}=\left(\frac{g_{t}\left(h_{t} i_{0}+N_{\mathrm{S}}\right)}{g_{r}\left(h_{r} i_{0}+N_{\mathrm{S}}\right)}\right)^{\gamma}$.

Neglecting noise terms, the naive gain factor $a_{n}$ is given by:

$a_{n}=\left(\frac{g_{t} h_{t}}{g_{r} h_{r}}\right)^{\gamma}$.

Both with changes in gain $g$ and amount of light $h$, the effect on the naive gain factor $a_{n}$ is the same, see (6).

\section{Proposed Algorithms}

To correct for changing intensity we need to estimate the apparent gain factor $a$ between two images $i_{r}$ and $i_{t}$ defined

\footnotetext{
${ }^{1}$ Only the characteristics of the CCD sensor have been modeled, not the optical effects such as vignetting.
} 
in Sect. 3.1. To obtain constant intensity over time we compare all images to the reference image $i_{r}$. This results in the apparent gain factor between the reference image and each other image. These apparent gain factors will be used to correct the corresponding images.

\subsection{Estimation of the Intensity Factor}

We intend to give an accurate non-iterative estimate of the apparent gain factor $a$. Because of the noise in both images, this parameter estimation problem is not trivial. Also, the less computation an algorithm requires, the easier it can be implemented in real-time. Therefore we also present simplifications to the theoretically optimal algorithm. In Sect. 5 we will experimentally compare these algorithms.

As the majority of the noise contributions are signal dependent, a Weighted Least Squares (WLS) estimate is a theoretically optimal non-iterative estimate. Minimizing the criterion

$L_{2}=\sum_{s \in S} w_{s}^{2}\left(i_{t, s}-a i_{r, s}\right)^{2}$,

for all pixels $s$ in set $S$ in least squares sense gives

$a_{\mathrm{WLS}}=\frac{\sum_{s \in S} w_{s}^{2} i_{r, s} i_{t, s}}{\sum_{s \in S} w_{s}^{2} i_{r, s}^{2}}$.

The weights $w$ can be calculated using the inverse of the image noise, which depends on the intensity. The general formula for a non-unity $\gamma$ is quite complicated, and here is approximated by the case for unity $\gamma$. Assuming shot noise, the noise variance of $i_{t}$ will relate to $i_{t}$, and similar the noise variance of $i_{r}$ will relate to $i_{r}$. This leads to the following weights

$w_{s}^{2}=\frac{i_{r, s}}{i_{t, s} i_{r, s}+i_{t, s}^{2}}$,

using $a=\frac{i_{t, s}}{i_{r, s}}$, as follows from (1), in the weights. This will however increase the influence of low intensity values. For these values the simplification used is not valid, as the amount of additive noise will be larger than the amount of signal dependent noise.

To reduce the influence of low intensities and at the same time reduce computational requirements, we can use equal weights. This gives us the standard Least Squares (LS) estimate

$a_{\mathrm{LS}}=\frac{\sum_{s \in S} i_{r, s} i_{t, s}}{\sum_{s \in S} i_{r, S}^{2}}$.

Even simpler and requiring fewer computations is using only the Quotient of the Average (QofA), related to the $L_{1}$ criterion:

$a_{\mathrm{QofA}}=\frac{\sum_{s \in S} i_{t, s}}{\sum_{s \in S} i_{r, s}}$.

All methods given above calculate the quotient of two numbers. For statistical outlier removal, see Sect. 4.2, it would be profitable to have an intensity ratio per pixel. This can also be useful for the extension to local intensity correction, see Withagen et al. (2008) Therefore, we also take the Average of the pixel-wise Quotient (AofQ) into account

$a_{\mathrm{AofQ}}=\frac{1}{|S|} \sum_{s \in S} \frac{i_{t, s}}{i_{r, s}}$,

with $|S|$ the number of pixels in the set of pixels $S$.

The experiments will show that it is important to be robust against outliers. Also, the quotient between two noisy images typically has a positively skewed distribution (Wikipedia on Ratio distribution) where the mean would over-estimated the apparent gain factor. For these reasons the median is also taken into account

$a_{\mathrm{QofM}}=\frac{\mathrm{M}_{s \in S} i_{t, s}}{\mathrm{M}_{s \in S} i_{r, s}}$,

and

$a_{\mathrm{MofQ}}=\mathrm{M}_{s \in S} \frac{i_{t, s}}{i_{r, s}}$,

where $\mathrm{M}$ denotes the median of a set of numbers.

\subsection{Outlier Removal Algorithm}

Comparing the two images $i_{r}$ and $i_{t}$ we should take into account that not all pixels will be stationary in dynamic scenes. Pixels depicting dynamic scenes violate the assumption of equal scene introduced in Sect. 3.1. The equations given above are thus only valid for pixels depicting stationary scene. Non-stationary pixels should be excluded from the apparent gain factor estimation.

We will investigate outlier removal based on statistics. For applications using foreground/background classification, moving objects will cause outliers. In such case classification between foreground and background can be used for outlier removal. However, this makes the global intensity correction algorithm less general.

Statistical outlier removal is based on the observation that the majority of the pixels depict the same scene in both images. We calculate the average $\mu_{q}$ and standard deviation $\sigma_{q}$ of the pixel-wise ratio $q_{s}$ between the two images. Pixels for which $\left|q_{s}-\mu_{q}\right|<T_{\text {outlier }} \sigma_{q}$ holds are labelled as outlier. Different values of $T_{\text {outlier }}$ have not been evaluated, but it should be of minor influence as long as it is chosen such 
that enough pixels remain for a statistically accurate estimate, but that only those pixels remain that are very unlikely to be outliers. We will use $T_{\text {outlier }}=1$.

Another set of pixels that should not be taken into account are pixels that are close to either the upper or lower bound of the range of pixel values. Pixels close to the upper bound may suffer from saturation problems. For pixels close to the lower bound the additive noise and dark current cannot be neglected, and also the influence of the shot noise, with a $\sqrt{i}$ relationship, will be higher. For the low values this may lead to a more biased estimates. Therefore, we will ignore pixels within 10 percent of the upper and lower bound. Again, varying these numbers is expected to have minor influence on the results.

\subsection{Creation of the Reference Image}

All algorithms proposed need a reference image to compare the current image to. In our experiments, we will use the first image of the image sequences as reference image. This is the most general solution and requires the least amount of computations. For most applications however, the reference image should be updated as the scene might change. Some alternatives will be discussed below.

The reference image can be periodically renewed by selecting a new image from the input. The image can be selected at random, or based on the amount of background it contains. The latter is preferred as it will contain less moving objects.

When using background modelling (Priebe 1994; Stauffer and Grimson 2000) it is possible to use the background model as reference. With a Gaussian mixture model, the average of the kernel with the largest weight can be used to compare the current image to, but it is also possible to use the mean of the best fitting kernel. The latter is expected to give better results for multi-modal backgrounds. Compared to choosing an image from the sequence, we expect the amount of noise and the number of foreground pixels in the EM-model reference image to be lower. An additional advantage of using the EM-model to create the reference image is that it is always up-to-date. When the background changes, the reference image is automatically adapted. Drawbacks are a small amount of additional computation.

For a slowly moving camera, each image can be compared to the previous image. If the motion is (approximately) known, only the overlapping area between the images should be used for apparent gain factor estimation.

\section{Experimental Evaluation}

We will experimentally evaluate the proposed algorithms. We will use simulated images to evaluate the accuracy and usability of the different intensity estimation algorithms in Sect. 5.1. We compare the algorithms to each other and to ground truth. Also, the need for outlier removal is evaluated. The effect of intensity correction in conjunction with classification between foreground and background on real images is demonstrated in Sect. 5.2. We look into the runtime of the algorithms in Sect. 5.3. A discussion of experimental results is given in Sect. 5.4.

For the evaluation of the usability we demonstrate intensity correction in conjunction with foreground/background classification. For each image the apparent gain factor is calculated and the image is corrected for it. With this corrected image, a model of the background is updated using the online Expectation Maximization (EM) algorithm (Priebe 1994). Four kernels are used to model the background. They are updated with an update speed $u_{\mathrm{B}}$.

The classification algorithm proposed by Stauffer (2000) is used to do classification between foreground and background. It is based on the assumption that the color distributions of the foreground and background are distinct. This way, separate kernels will be used to model the foreground and background. Each kernel is assigned a label, determining whether it models foreground or background. The background kernels together describe more then a fraction $F$ of all data. We use $F=0.5$ as proposed by Stauffer. Pixel classification is performed by determining whether a pixel can be assigned to any of the kernels labelled as background. A pixel is assigned to a kernel if it's value is within $T_{\text {Stauffer }}$ times the kernels standard deviation from the kernel mean (Stauffer proposes $T_{\text {Stauffer }}=2.5$ in Stauffer and Grimson 2000).

\subsection{Comparison by Simulation}

We will evaluate the proposed algorithms using a simulated image sequence. For each image the apparent gain factor $a$ is estimated and the image is corrected according to (1). The corrected image is used to measure the performance of the intensity correction algorithm and to perform foreground/background classification.

\subsubsection{Image Generation}

Images are generated based on the full model of the CCD given by (19), so without simplifications. As scene we use the red channel of the first image from the Intratuin image sequence, see Fig. 1. In each frame $20 \%$ of the pixels is selected at random and they are given a foreground value. The foreground value is drawn from a uniform distribution between 0 and 0.5 , while the distribution of the background lies between 0 and 1 with peaks around 0.3, 0.6 and 0.8 , see Fig. 1 for a noiseless example image and its histogram. We choose the parameter settings of the image simulation 


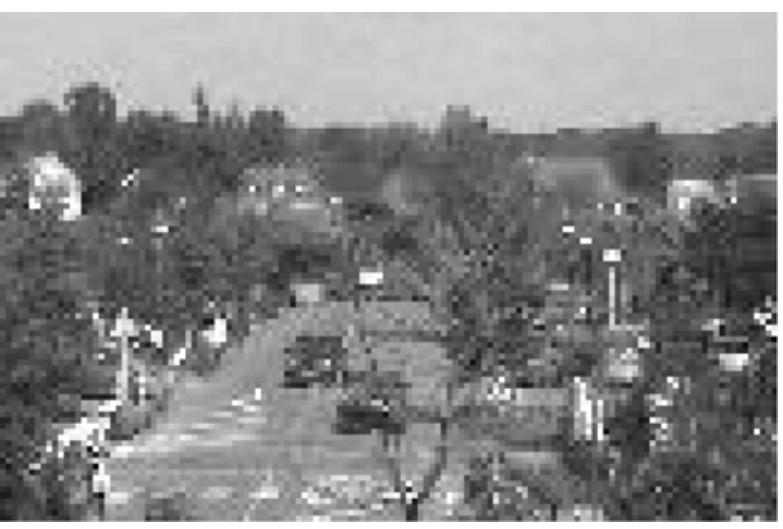

(a) Simulation image

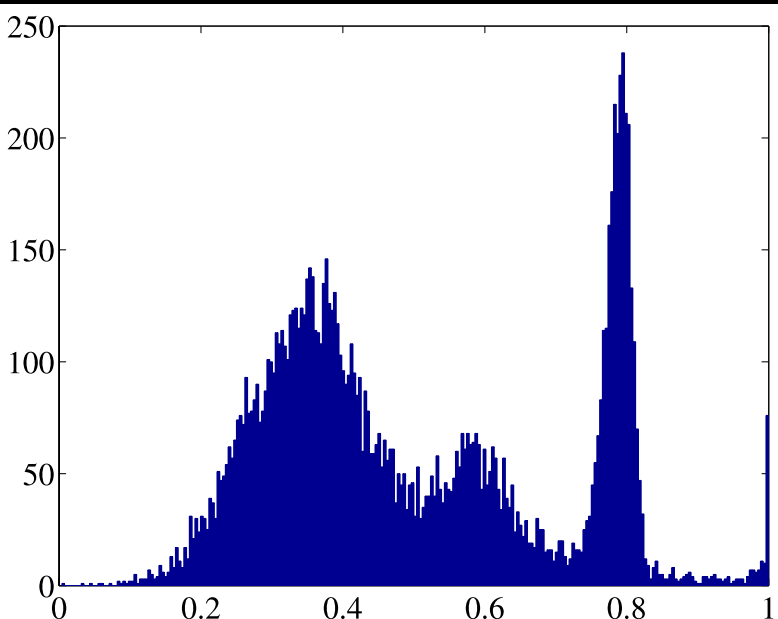

(b) Histogram of simulation image

Fig. 1 Simulation image and its histogram. The image on the left shows an example image used for the simulation experiments. On the right the histogram of this image is given

Fig. 2 The value of the camera gain for the simulated images

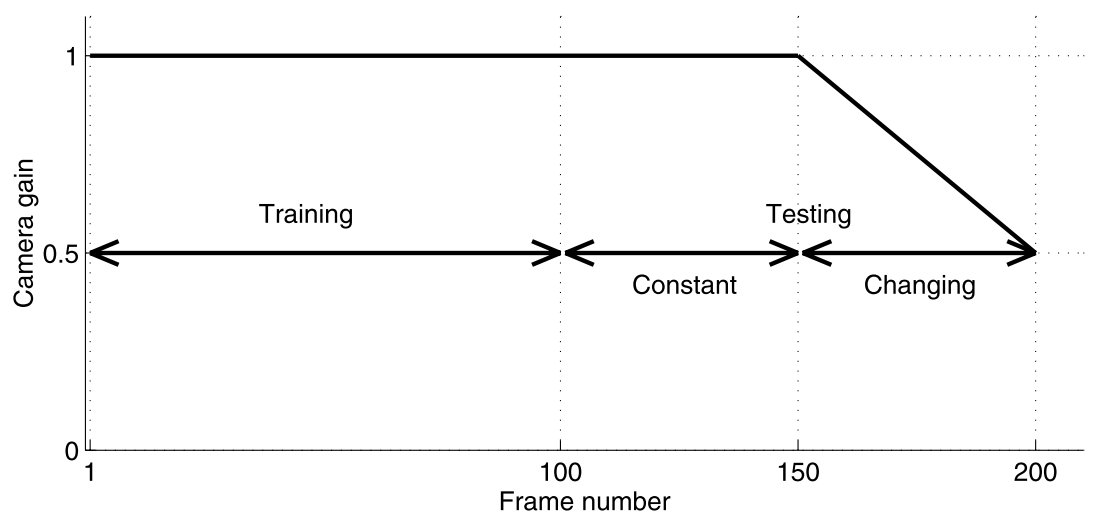

such that they approach an average camera from the cameras characterization in Withagen et al. (2007).

The scene intensity is kept constant $h_{t}=h_{r}$ and the camera gain $g_{t}$ is varied, see Fig. 2. The camera gain is 1 for images 1 to 150 for training and evaluation of the algorithms on images without changes in intensity. The intensity linearly decreases from 1 at image 150 to 0.5 at image 200 for the evaluation of the algorithms on images with a changing intensity.

Besides the image sequence $i_{t}$, two additional images are created. The reference image $i_{r}$ which is used for the estimation of the apparent gain factor together with the current image, and the ground truth image $i_{\mathrm{gt}}$. These images have equal illumination and camera gain and do not have foreground pixels. The ground truth image is noiseless, the reference image contains noise.

\subsubsection{Evaluation Criteria}

Two criteria for evaluation are used, one for the accuracy of the apparent gain factor and one for the usability in conjunction with foreground/background classification.
For accuracy evaluation we use the root mean squares error between the intensity corrected current image and the ground truth image for all pixels depicting background:

$e_{\text {accuracy }}=\sqrt{\frac{1}{|B|} \sum_{b \in B}\left(i_{\mathrm{gt}, b}-\frac{1}{a} i_{t, b}\right)^{2}}$,

with $|B|$ the number of pixels in the set of background pixels $B$. Note that as we use a realistic setting with noise in the current image, this criterion gives for perfect intensity correction the standard deviation of the image noise.

The usability of the intensity correction algorithm is evaluated with foreground/background classification. A Mixture of Gaussians model is updated using online Expectation Maximization, with an update speed $u_{\mathrm{B}}=0.05$. With this model, classification is performed using the algorithm of Stauffer with a threshold $T_{\text {Stauffer }}=3$. These settings were found to be optimal for the reference method, without intensity correction, and the given evaluation criterion for usability defined below. Evaluation is started after 100 frames, 
Table 1 Accuracy results for simulated data. Shown is the average of the error $e_{\text {accuracy }}$ in percentages (lower is better). The standard deviations over the frames are given between brackets

\begin{tabular}{llllll}
\hline Outl. rem.: & \multicolumn{2}{l}{ Static intensity } & & \multicolumn{2}{l}{ Changing intensity } \\
\cline { 5 - 6 } & No & Yes & & No & Yes \\
\hline Method: & & & & $12.56(7.28)$ & \\
No & $1.261(0.009)$ & & $1.2660(0.008)$ & \\
GT & $1.261(0.009)$ & & $2.631(0.076)$ & $1.268(0.008)$ \\
QofA & $2.640(0.094)$ & $1.2628(0.009)$ & & $2.319(0.169)$ & $1.307(0.047)$ \\
QofM & $2.402(0.183)$ & $1.2854(0.038)$ & & $1.361(0.072)$ & $1.267(0.008)$ \\
AofQ & $2.361(0.083)$ & $1.2619(0.009)$ & & $1.277(0.008)$ & $1.266(0.008)$ \\
MofQ & $1.263(0.011)$ & $1.2606(0.009)$ & & & $1.269(0.008)$ \\
LS & $2.897(0.107)$ & $1.2637(0.009)$ & & $2.878(0.084)$ & $1.276(0.009)$ \\
WLS & $7.496(0.410)$ & $1.2714(0.009)$ & & $7.199(0.354)$ & \\
\hline
\end{tabular}

Table 2 Usability results for simulated data. Shown is the average of the error $e_{\text {usability }}$ in percentages (lower is better). The standard deviations over the frames are given between brackets. Note that these results cannot be compared to the results on real data as only one color has been used in the simulation

\begin{tabular}{lccccc}
\hline Outl. rem.: & \multicolumn{2}{l}{ Static intensity } & & \multicolumn{2}{l}{ Changing intensity } \\
\cline { 2 - 3 } \cline { 5 - 6 } & No & Yes & & No \\
\hline Method: & & & & \\
No & $6.822(0.716)$ & & $6.87(5.16)$ & \\
GT & $6.822(0.716)$ & & $6.699(0.664)$ & \\
QofA & $8.096(1.162)$ & $6.813(0.708)$ & $6.887(0.713)$ & $6.706(0.665)$ \\
QofM & $7.894(1.066)$ & $6.876(0.694)$ & $6.873(0.716)$ & $6.760(0.669)$ \\
AofQ & $7.751(1.027)$ & $6.817(0.711)$ & $6.828(0.706)$ & $6.705(0.665)$ \\
MofQ & $6.823(0.717)$ & $6.822(0.716)$ & $6.710(0.652)$ & $6.710(0.661)$ \\
LS & $8.403(1.335)$ & $6.819(0.705)$ & $6.937(0.714)$ & $6.705(0.664)$ \\
WLS & $18.11(6.38)$ & $6.820(0.708)$ & $7.927(1.102)$ & $6.709(0.664)$ \\
\hline
\end{tabular}

allowing the EM background model to learn on the first 100 frames.

The impact of the intensity correction for our application is expressed in a usability measure: the classification performance. It shows whether using intensity correction gives an improvement in the results. We define the usability of the intensity correction as the fraction of erroneously classified pixels

$e_{\text {usability }}=\frac{N_{\text {erroneous }}}{N_{\text {total }}}$,

with $N_{\text {erroneous }}$ the number of erroneously classified pixels and $N_{\text {total }}$ the total number of pixels. This assumes equal cost for missed foreground pixels and erroneously detected foreground pixels.

\subsubsection{Simulation Results}

Besides the six proposed methods, correcting with the ground truth $a$ (GT) and using the uncorrected image (No) are also presented. The different algorithms for estimating the apparent gain factor are evaluated with and without outlier removal. For all results, the mean and standard deviation over the images were calculated for the two sections: static and changing intensity. The results shown are averaged over fourteen independent noise realizations.

The results for all combinations are given in Tables 1 and 2. Most important observations are a ten times lower accuracy error and a three times lower usability error for methods using outlier correction compared to no correction. Methods without outlier correction perform significantly worse.

From the accuracy results we see that as long as there is some kind of outlier robustness (statistical outlier removal or methods based on the median), accuracy is for most combinations close to the image noise. Exception is the method QofM, which performs slightly worse. For changing intensity, the error with correction is ten times lower than without correction. Without outlier removal results are significantly worse, even on the section with constant intensity.

For usability the results are even more consistent. All combinations that are robust to outliers (statistical outlier removal or median) give errors close to the error of correcting with the ground truth. The error of $6.8 \%$ is caused by the fraction of the $20 \%$ foreground pixels that overlap in color with the scene. This should be compared to no correction, where the error triples in dynamic situations. Com- 


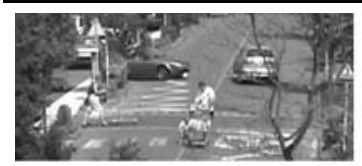

(a) 500

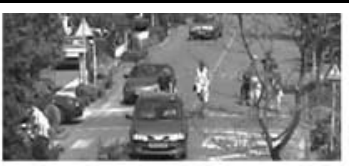

(b) 750

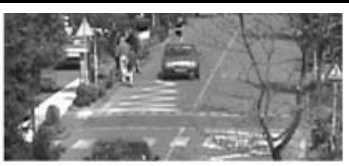

(c) 1000

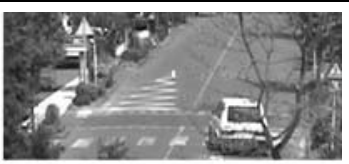

(d) 1250

Fig. 3 Some images from Intratuin sequence

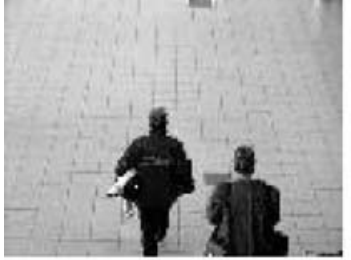

(a) 250

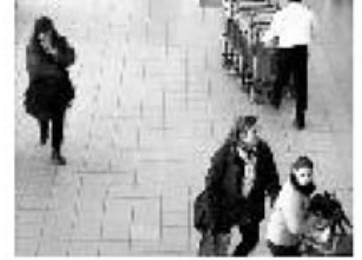

(b) 750

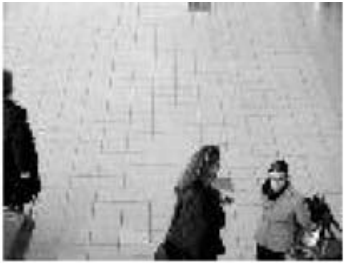

(c) 1250

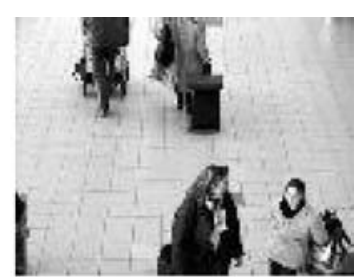

(d) 1750

Fig. 4 Some images from Schiphol sequence

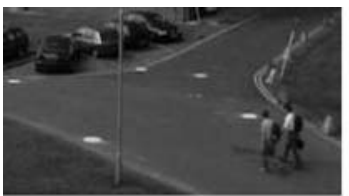

(a) 2000

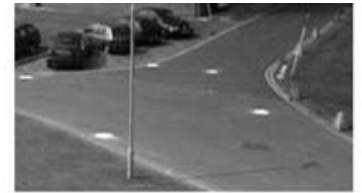

(b) 3500

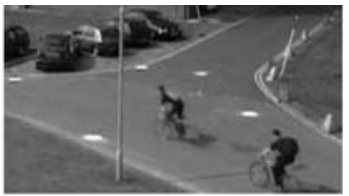

(c) 4500

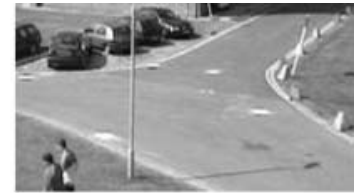

(d) 5500

Fig. 5 Some images from PETS sequence

binations without outlier removal perform again worse than those with

As could be expected the WLS method is only optimal when its assumptions are fulfilled, in particular with respect to the probability density distributions, so without outliers. The method is extremely sensitive to outliers, shown by the results without outlier removal. Even the few outliers remaining after outlier removal are sufficient to decrease the performance of the WLS method. To a lesser extend, the same holds for LS. A solution would be to use robust iterative estimators, like M-estimators, but those are very timeconsuming and difficult, if not impossible, to use in realtime. The difference in performance between LS and WLS suggests that an additive noise model (LS) gives in this case a better description of the data than the signal dependent noise model used in WLS.

\subsection{Evaluation Using Real Image Sequences}

In simulation experiments optimal parameter settings for the background classification algorithm can be used. In practical situations these optimal settings are often unknown, and the system will operate in general at sub-optimal settings of these parameters. So the sensitivity of the performance to suboptimal settings will play a major role in the evaluation on real image sequences.

\subsubsection{Image Sequences}

Three image sequences were used for the evaluation, see also Figs. 3, 4 and 5:

- Intratuin: Parking lot with waving tree branches. In this sequence there is no significant variation in intensity. The sequence contains cars and pedestrians, moving both slowly and fast. This sequence has $150 \times 350$ pixels and 1250 frames.

- Schiphol: Recorded in the main hall of Schiphol airport. There are a lot of global intensity variations due to automatic gain control. The sequence contains relatively large objects, some of which become stationary. This sequence has $90 \times 120$ pixels and 1750 frames.

- PETS 2001: We used a cut-out from the images of dataset 3, training, camera 1 from the IEEE International Workshop on Performance Evaluation of Tracking and Surveillance 2001 (PETS). There are large local changes in illumination intensity due to clouds. The images contain relatively few object pixels. The part of the image that is used is between rows 300 and 520, skipping the odd rows and between columns 350 and 750, skipping the odd columns. This sequence has $120 \times 200$ pixels and 5500 frames.

All sequences are RGB color video data with eight bit per color. For each sequence, five to eleven images were man- 
ually labeled. Each pixel was labeled: foreground, background or any. The label any is used for the edges of objects, where it is difficult (for a human) to decide whether this pixel should be labeled as either foreground or background. It is also used for some artifacts in the images like moving objects that stop moving.

The Intratuin and Schiphol sequences are recorded by us and they are available through our website [www.science.uva $\mathrm{nl} /$ sites/PedestrainClass/paul/] together with the manually labeled ground truth for all sequences. The PETS 2001 data is available through pets2001.visualsurveillance.org.

\subsubsection{ROC Performance}

We evaluate the intensity correction algorithms based on their usability in conjunction with classification between foreground and background. The images are corrected with each of the proposed intensity correction algorithms after which the background model is updated and foreground/background classification is performed.

The choice of the best algorithm for foreground/background classification depends on the application. In order to make a good choice, the ratio of the cost of a false alarm and the cost of a missed detection must be known. For the experiments on simulated images described in Sect. 5.1, unity cost was assumed.

When comparing different classification algorithms, the parameter settings of all algorithms should be optimized for the chosen cost ratio. For a different application, a different ratio might be in use and therefore a different algorithm might be optimal. It would be efficient to compare for different cost ratios at once.

Often, Receiver Operator Characteristics (ROCs) are used for this. We construct a ROC curve by computing the convex hull of the foreground/background classification results for a selection of parameter settings, see Provost and Fawcett (2001), Scott et al. (1998). Given a certain cost fraction, the optimal method can now easily be found in the graph. We call this curve the total-ROC.

Before foreground/background classification, the EM model was initialized by updating the model for images 500,499 , and so on until image 1 using a constant update speed $u_{\mathrm{B}}=0.05$. As the first frame on which we evaluate is frame 500, this allows the algorithm to initialize during 1000 frames. This enables the evaluation of very low update speeds, for which the sequences would not be long enough to obtain a converged model of the background. The entire image sequence was processed several times, each time using different values for the update speed $u_{\mathrm{B}}$ and threshold $T_{\text {Stauffer. }}$.

In Fig. 6 total-ROC curves for a number of methods are given. These are the convex hulls of the results of experiments in which both the update speed $u_{\mathrm{B}}$ and the classification threshold $T_{\text {Stauffer }}$ of the background modelling and classification algorithm are varied. As most methods coincide, we selected only a few methods: the reference method No correction, MofQ with and without outlier removal and QofA with outlier removal. For the PETS013TR1 and Schiphol sequences the improvement with any of the proposed methods is significant, for the Intratuin sequence there is also a slight improvement.

Table 3 gives an overview of the surface above the ROC for all methods. For Intratuin, most methods that are robust to outliers, i.e. statistical outlier removal or using the median, perform slightly better than without global intensity correction. The only exception is QofM without outlier removal. For Schiphol and PETS01-3TR1 the methods with outlier removal perform significantly better with an error reduction of a factor two and three respectively. Best performance is obtained using MofQ without statistical outlier removal.

\subsubsection{Parameter Invariant Performance Evaluation (PIPE)}

Unfortunately, the total-ROC is in this case not a sufficient criterion. The problem is that the effect of global intensity differences can be partially solved by faster updating of the background, at the cost of robustness. A method might perform well on one image sequence with a certain update speed, but this does not mean it will perform that well with equal update speed on another set of images, recorded under different conditions. This is a matter of robustness against setting the parameters, specifically the update speed, that is not taken into account by comparing total-ROCs.

We therefore propose the Parameter Invariant Performance Evaluation (PIPE) criterion. PIPE is a measure that gives in one number the average performance on an image sequence and the robustness against changing the parameters to the optimal parameter settings for other image sequences. A low error can be achieved by a method that performs well with all parameter settings, or one that has optimal performance for one setting regardless of the image sequence. Both cases are attractive to use in practice, where it is difficult and impractical to tune parameters as the circumstances change.

PIPE is based on ROCs. The parameters we intend to vary are the threshold $T_{\text {Stauffer }}$ and the update speed $u_{\mathrm{B}}$, where the update speed is the parameter we wish to be robust against. We first analyze the effect of this parameter. Therefore, we create for each image sequence $l_{\text {seq }}$ and update speed $u_{\mathrm{B}}$ a $u_{\mathrm{B}}$-ROC by varying threshold $T_{\text {Stauffer }}$ only. We calculate area $A\left(l_{\text {seq }}, u_{\mathrm{B}}\right)$ under this $u_{\mathrm{B}}-\mathrm{ROC}$. This area lies between zero and one, where one corresponds to the perfect classification result and a value of 0.5 can be obtained by classifying at random.

Figure 7 shows the areas $A\left(l_{\text {seq }}, u_{\mathrm{B}}\right)$ under the $u_{\mathrm{B}}$-ROC for different update speeds for the methods selected before. 
Fig. 6 Total-ROCs of the classification results on real data with different values of update speed and threshold. Only a selection of the methods is shown as most methods coincide
Table 3 ROC results for real image sequences. Given is the average of the surface above the total-ROC in percentages (lower is better)

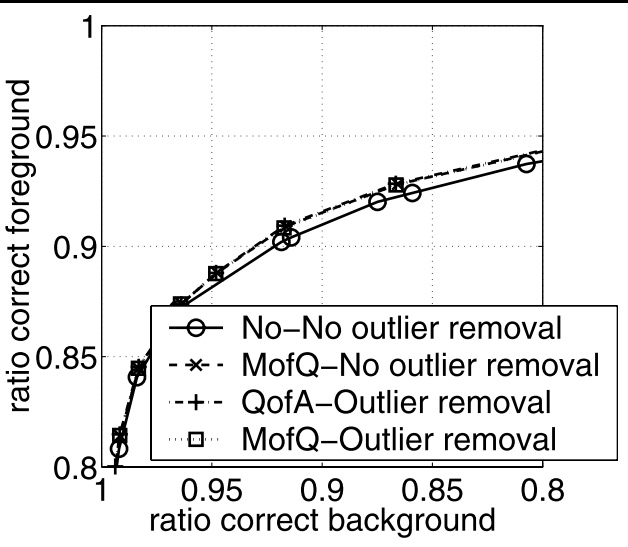

(a) Intratuin

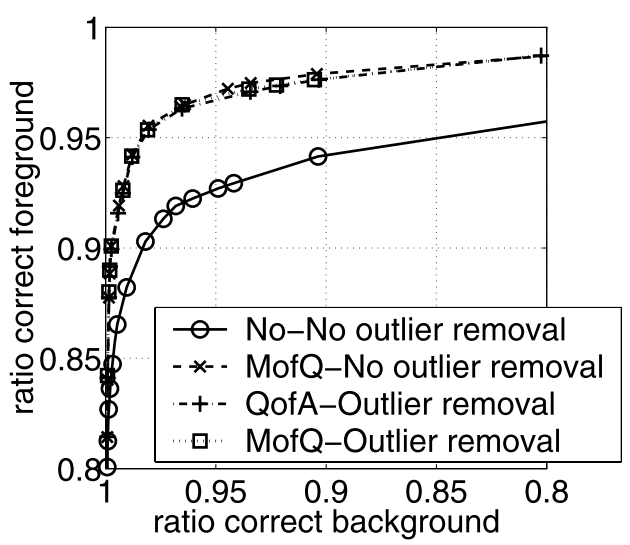

(c) PETS01-3TR1

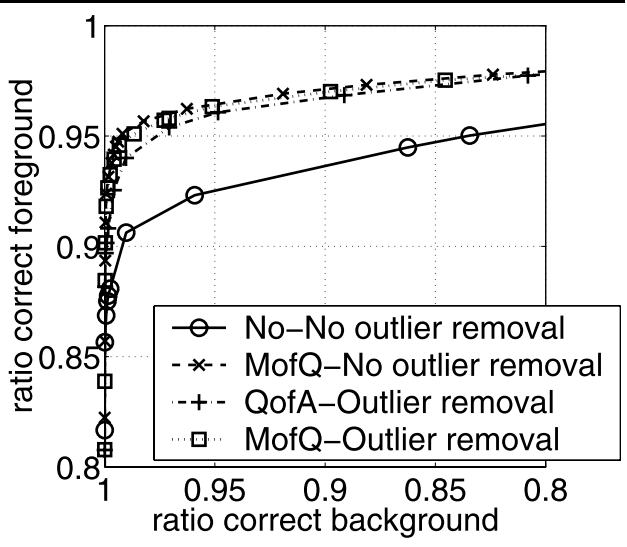

(b) Schiphol

\begin{tabular}{|c|c|c|c|c|c|c|c|c|}
\hline \multirow{2}{*}{$\begin{array}{l}\text { Data: } \\
\text { Outlier removal: }\end{array}$} & \multicolumn{2}{|c|}{ Intratuin } & \multicolumn{2}{|c|}{ Schiphol } & \multicolumn{2}{|c|}{ PETS01-3TR1 } & \multicolumn{2}{|c|}{ Average } \\
\hline & No & Yes & No & Yes & No & Yes & No & Yes \\
\hline \multicolumn{9}{|l|}{ Method: } \\
\hline No & 3.95 & & 2.59 & & 2.28 & & 2.94 & \\
\hline QofA & 4.19 & 3.67 & 2.71 & 1.38 & 0.80 & 0.81 & 2.57 & 1.95 \\
\hline QofM & 5.38 & 3.71 & 1.35 & 1.34 & 0.82 & 0.80 & 2.51 & 1.95 \\
\hline AofQ & 4.21 & 3.68 & 2.03 & 1.35 & 0.82 & 0.79 & 2.35 & 1.94 \\
\hline MofQ & 3.68 & 3.68 & 1.25 & 1.32 & 0.76 & 0.78 & 1.90 & 1.93 \\
\hline LS & 4.44 & 3.69 & 3.01 & 1.42 & 0.81 & 0.80 & 2.75 & 1.97 \\
\hline WLS & 7.67 & 3.68 & 6.08 & 1.60 & 1.16 & 0.80 & 4.97 & 2.03 \\
\hline
\end{tabular}

This immediately shows that different image sequences can require different parameter settings, but that intensity correction makes the algorithm more robust against setting of the update speed. As a consequence, intensity correction makes the algorithm more robust against changes of the environment. The figure also shows that intensity correction cannot be substituted by faster updating of the background model. For the PETS01-3TR1 sequence this might be a solution, but for the Intratuin and Schiphol sequences performance significantly drops for higher update speeds due to misclassification of slowly moving objects.
We wish to obtain one error measure for each image sequence. This can be achieved by averaging the results of the $u_{\mathrm{B}}$-ROC over the different values of the update speed. In order to introduce robustness into the evaluation criterium, we weight the different contributions. The weight is determined by the number of times this update speed was optimal for any of the image sequences. A value of the update speed is optimal when it lies on the convex hull of the total-ROC. $U\left(u_{\mathrm{B}}\right)$ is the average over all image sequences of the normalized histograms of the occurrence frequency of update speeds on the convex hulls of the total-ROCs. 
Fig. 7 Each legend entry corresponds to two lines in each figure. The curved line is the area under the $u_{\mathrm{B}}$-ROC for different values of the update speed. The horizontal lines indicates the area under the total-ROC. Only a selection of the methods is shown as most methods coincide. Figure (d) shows the corresponding $U\left(u_{\mathrm{B}}\right)$ histograms

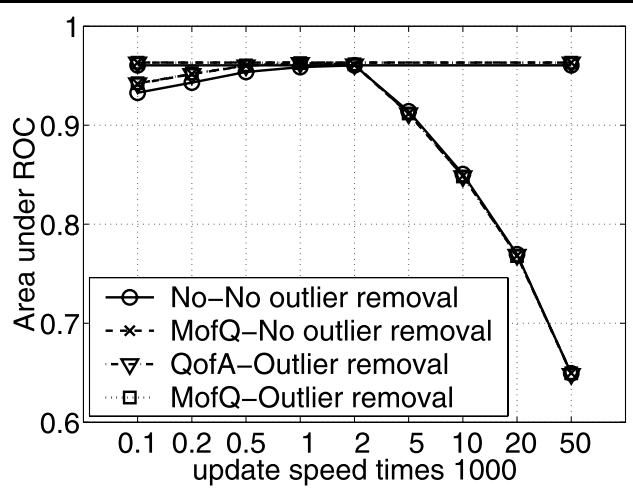

(a) Intratuin

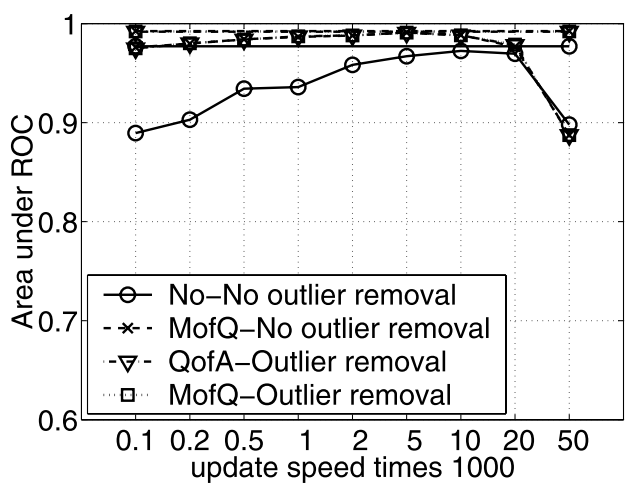

(c) PETS01-3TR1

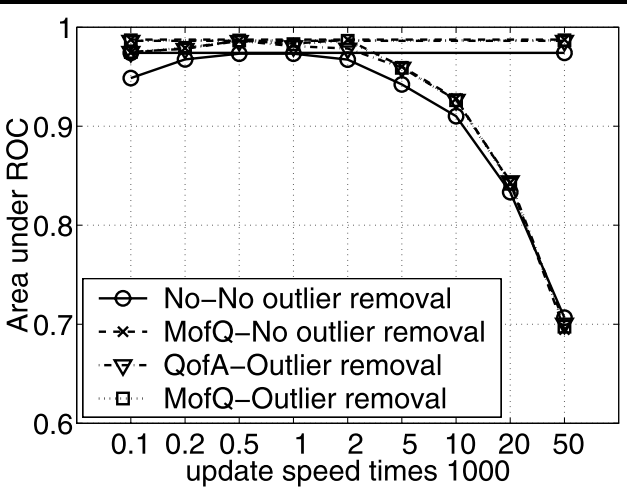

(b) Schiphol

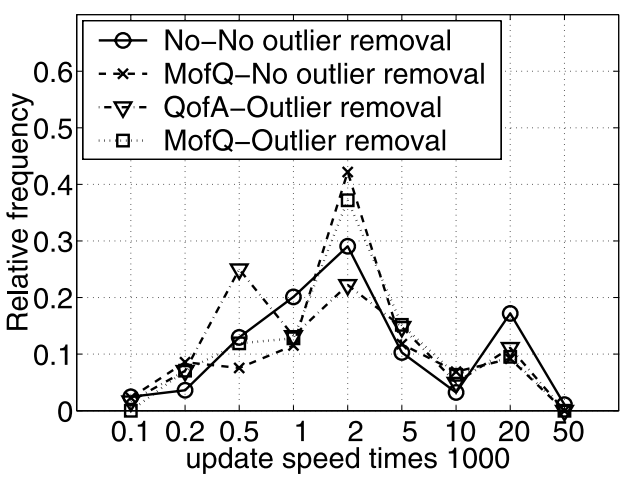

(d) $U(u)$ histogram
Table 4 PIPE results for real image sequences. Given is the average of the error $e_{\mathrm{PIPE}}$ in percentages (lower is better)

\begin{tabular}{|c|c|c|c|c|c|c|c|c|}
\hline \multirow{2}{*}{$\begin{array}{l}\text { Data: } \\
\text { Outlier removal: }\end{array}$} & \multicolumn{2}{|c|}{ Intratuin } & \multicolumn{2}{|c|}{ Schiphol } & \multicolumn{2}{|c|}{ PETS01-3TR1 } & \multicolumn{2}{|c|}{ Average } \\
\hline & No & $\overline{\text { Yes }}$ & No & Yes & No & Yes & $\mathrm{No}$ & Yes \\
\hline \multicolumn{9}{|l|}{ Method: } \\
\hline No & 8.66 & & 6.15 & & 5.04 & & 6.61 & \\
\hline QofA & 7.92 & 7.39 & 5.13 & 3.97 & 1.53 & 1.46 & 4.86 & 4.27 \\
\hline QofM & 8.96 & 7.54 & 3.83 & 3.90 & 1.39 & 1.41 & 4.73 & 4.28 \\
\hline AofQ & 7.94 & 7.39 & 4.92 & 3.83 & 1.48 & 1.47 & 4.78 & 4.23 \\
\hline MofQ & 7.14 & 7.23 & 3.47 & 3.63 & 1.41 & 1.35 & 4.01 & 4.07 \\
\hline LS & 8.50 & 7.67 & 5.38 & 4.16 & 1.45 & 1.45 & 5.11 & 4.43 \\
\hline WLS & 12.32 & 7.90 & 10.17 & 4.44 & 1.76 & 1.49 & 8.08 & 4.61 \\
\hline
\end{tabular}

Our Parameter Invariant Performance Evaluation error $e_{\text {PIPE }}$ is now given by one minus this weighted average

$e_{\mathrm{PIPE}}(l)=1-\frac{1}{N_{u_{\mathrm{B}}}} \sum_{u \in n_{u_{\mathrm{B}}}} U\left(u_{\mathrm{B}}\right) A\left(l, u_{\mathrm{B}}\right)$,

with $n_{u_{\mathrm{B}}}$ the list of $N_{u_{\mathrm{B}}}$ different values of $u_{\mathrm{B}}$ that are used.

\subsubsection{PIPE Results}

Full results according to $e_{\text {PIPE }}$ described above are given in Table 4. Error reductions of almost a factor two on the Schiphol sequence and almost a factor four on the PETS013TR1 sequence are obtained. Below we will discuss the results for all sequences in more detail.
For the Intratuin sequence, results of combinations that either do statistical outlier removal or are based on the median are slightly better then the result without correction, except for QofM without statistical outlier removal. It is not surprising that the results are close to that without correction as this image sequence does not contain significant intensity variations. However, this data does show that the use of intensity correction does not make results worse when there is no need for correction. Methods that perform best are AofQ and QofA with statistical outlier removal and MofQ.

The Schiphol sequence contains considerable changes in intensity due to automatic gain control. The error is reduced by approximately a factor one-and-a-half for methods that 
Table 5 The runtime of the different methods. Times are in milliseconds, standard deviation is lower than $1.0 \mathrm{~ms}$ for all measurements

\begin{tabular}{lccccrc}
\hline Method: & WLS & LS & QofA & QofM & AofQ & MofQ \\
\hline No outlier removoval & 36.1 & 10.2 & 2.5 & 52.0 & 6.1 & 30.6 \\
Statistical outlier removoval & 73.1 & 46.9 & 39.4 & 89.1 & 38.3 & 62.7 \\
\hline
\end{tabular}

are robust to outliers. Non-robust methods results are significantly worse. Best performance is obtained using MofQ.

The PETS01-3TR1 sequence contains changes in intensity caused by moving clouds. Therefore, these changes are not always global. Nevertheless, results improve significantly when using any of the proposed global intensity correction algorithms. An error reduction of almost a factor four is obtained, the methods based on the median perform best on this image sequence. Outlier removal does not seem necessary for the LS method on this image sequence. This is because compared to the other data, there are much less outliers in this image sequence as only a small fraction of the pixels depict foreground. In this case outlier removal still reduces the number of pixels and consequently the estimation accuracy of the apparent gain factor.

On average, outlier robustness is essential for good results. MofQ performs best with an average error reduction of more than a factor 1.6.

\subsection{Runtime}

Our goal is to find an intensity correction algorithm to be used as pre-processing for real-time applications. Therefore, runtime is an important issue.

We ran all algorithms on an image of 100,000 pixels. The runtime of each algorithm was averaged over 100 runs. The computations were performed on a Pentium IV $2500 \mathrm{MHz}$ processor under Windows 2000 Professional. The algorithms were implemented in Matlab 6.1 www.mathworks.com. The Matlab implementations for the computation of the mean and median were used and all computations were performed in double precision. On other platforms results may differ, but the ordering of methods complexity is assumed to be similar.

The results are given in Table 5. This table shows that when no statistical outlier removal is necessary, QofA and AofQ are most affordable, followed by LS. These methods are a good choice in conjunction with other ways of outlier removal like foreground/background classification.

Otherwise statistical outlier removal should be used, except for methods based on the median as we have shown. We therefore compare the methods with a median without outlier removal to the other methods with statistical outlier removal. Then MofQ is the most affordable algorithm, followed by AofQ and QofA, all very close to each other.

It should be noted that it is not necessary to use all pixels in the image for intensity estimation. According to the amount of processing power available and the accuracy requested, a fraction of the available pixels can be used. Outlier removal also reduces the number of pixels that needs to be taken into account.

\subsection{Discussion of the Experiments}

According to simulation results, many algorithms seem to perform very well. Results on real images shall therefore be used to select between algorithms. We will look at the average ROC and PIPE performance over all image sequences. We then see that MofQ performs best, both with and without outlier removal. Without outlier removal, this is also the least computationally complex algorithm, considering that other methods require outlier removal. AofQ and QofA with statistical outlier removal are slightly more expensive, and their performance is also lower.

The use of WLS or LS is expensive and these methods are sensitive to remaining outliers. They are therefore not a good choice if perfect outlier removal cannot be guaranteed. Even though their average performance is good.

Both AofQ or MofQ use a per-pixel estimate of the gain factor. This allows for easy extension to local intensity correction. On the other hand, QofA and QofM have the advantage that they can also be used when the images cannot be compared pixel-wise, like in stereo vision, or when there is a small movement of the camera between the current image and the reference image.

In practice, the application requiring intensity correction should be considered before choosing a method. Considerations are the required accuracy, the available computing power, whether or not the camera is static and whether or not there is an outlier removal algorithm available. If images can be compared pixel-wise, MofQ is the best choice.

\section{Conclusions}

\subsection{Comparison to Previous Work}

Using simulation we have shown that the proposed intensity correction algorithms perform equally well as can be obtained using ground truth correction. Thus, under the assumptions of constant gamma and only global changes in intensity, there is no need to use more expensive algorithms like dynamic histogram warping (Cox et al. 1995), iterative weighted least squares estimation of the bias, gain and 
gamma (Tsin et al. 2001) and estimation of the gain and bias together with optic flow estimation (Altunbasak et al. 2003). Also, Cox et al. (1995) and Altunbasak et al. (2003) do not perform outlier removal. We have shown in this paper that robustness against outliers significantly improves accuracy and usability.

Our experiments on real data clearly show that only relying on the adaptation of the background modelling technique (McKenna et al. 2000; Toyama et al. 1999) is not an option. Tweaking the update speed to reach acceptable classification performance on one sequence immediately degrades the performance on other image sequences.

He et al. (2003) and Kamikura et al. (1998) estimate the intensity change between two images using the average and a least squares estimate respectively. However, these methods do not consider the effect of outliers, reducing accuracy and usability.

\subsection{Experimental Evaluation}

For the simulated images the RMS error between the corrected image and the ground truth image evaluates the accuracy. Results show that methods that are robust to outliers (either using statistical outlier removal or by using the median) performed very close to the noise level for image sequences without and with changes in intensity. This indicates that these methods are sufficiently accurate to be used in practical applications.

Usability of the different methods is demonstrated using background classification on the corrected image sequences. With constant image intensity, all methods that are robust to outliers obtained equal performance compared to using no correction. For image sequences with changing intensity these methods perform a factor ten better compared to no correction. This is similar to correcting with ground truth values.

ROC and PETS results on real images without temporal intensity changes show that most methods that are robust to outliers have slightly better classification performance as without intensity correction. Two image sequences with intensity changes show that the proposed methods cause significant improvement to the classification performance. Even with local intensity changes, significant improvements are shown. Error reduction of almost a factor two on the Schiphol sequence and more than a factor three on the PETS01-3TR1 sequence are obtained.

Using the proposed Parameter Invariant Performance Evaluation PIPE we have further shown that our proposed intensity correction introduces robustness against varying the update speed of the adaptive background modelling algorithm. It allows lower update speed to be used, preventing slowly moving objects to be incorporated in the background model.
The best method depends on the application. Overall, if images can be compared pixel-wise, the Median of the pixelwise Quotient of images, MofQ, is the best choice. It is robust to outliers and noise, and it is also a computationally efficient solution if it is compared to other methods with statistical outlier removal.

\subsection{General Conclusions}

In this paper is shown that using global intensity correction based on the ratio of pixels in conjunction with outlier robustness significantly improves background classification results. For image sequences not needing intensity correction, no decrease of performance is seen.

For the evaluation on real images the Parameter Invariant Performance Evaluation (PIPE) error measure, based on the ROC, is proposed. It combines both classification error and robustness against changes in parameter settings in one number.

It is believed that the proposed method can be applied and will provide similar benefit to other image processing algorithms based on Constant Image Brightness (CIB) or the Brightness Constancy Constraint Equation (BCCE).

Open Access This article is distributed under the terms of the Creative Commons Attribution Noncommercial License which permits any noncommercial use, distribution, and reproduction in any medium, provided the original author(s) and source are credited.

\section{Appendix A: Theoretical Model of a CCD Camera}

Healey (1994) describes the following model for a single pixel recorded at time $t$ using a CCD camera:

$i_{t}=g_{t}\left(h_{t} i_{0}+\mu_{\mathrm{DC}}+N_{\mathrm{S}}+N_{\mathrm{R}}\right)+N_{\mathrm{Q}}$,

with $h_{t} i_{0}$ the scene irradiance and $i_{t}$ the image intensity. The following noise contributions are present: ${ }^{2}$ the mean of the dark current $\mu_{\mathrm{DC}}$ is an offset, constant over time. The shot noise $N_{\mathrm{S}}$ has a Poisson distribution with $\mu_{\mathrm{S}}=0$ and $\sigma_{\mathrm{S}}$ depending on $i_{0}$. The readout noise $N_{\mathrm{R}}$ has a Gaussian distribution: $\mu_{\mathrm{R}}=0, \sigma_{\mathrm{R}}$ constant. The quantization noise $N_{\mathrm{Q}}$ has a uniform distribution $U\left(-\frac{q}{2}, \frac{q}{2}\right)$ with $q$ the smallest step in pixel value.

There are three ways to control the global image intensity, we will use the term apparent gain for their joint effect. It can be controlled using the camera gain $g_{t}$, or using camera shutter time or lens iris, modelled together using $h_{t}$. All can be fixed (manual control) or automatically adapted to the scene (automatic gain/shutter/iris control).

\footnotetext{
${ }^{2}$ We use $\mu_{x}$ for the mean of $x$ and $\sigma_{x}$ for its standard deviation.
} 
Additionally, most cameras apply a gamma adjustment to map the range of intensity values from the CCD to the available output range. Assuming it is implemented in the camera electronics just before digitization, (18) changes to

$i_{t}=g_{t}^{\gamma}\left(h_{t} i_{0}+\mu_{\mathrm{DC}}+N_{\mathrm{S}}+N_{\mathrm{R}}\right)^{\gamma}+N_{\mathrm{Q}}$,

with $\gamma$ the gamma value which is assumed to be time constant and equal for all pixels.

\section{A.1 Simplifications to the Model}

In Withagen et al. (2007) we report the experimental results of the different contributions in this CCD model. These results allow for simplification of the model. The simplifications are given in this subsection and lead to the model of the CCD camera to be used in the remainder of this paper.

Our experimental validation concludes that we can neglect the contribution of the dark current. This simplifies equation 19 to

$i_{t}=g_{t}^{\gamma}\left(h_{t} i_{0}+N_{\mathrm{S}}+N_{\mathrm{R}}\right)^{\gamma}+N_{\mathrm{Q}}$.

The remaining noise terms all are zero-mean. The shot noise $N_{\mathrm{S}}$ is signal dependent with $N_{\mathrm{S}}=O(\sqrt{i})$ and the readout noise $N_{\mathrm{R}}$ and the quantization noise $N_{\mathrm{Q}}$ are additive.

Our experiments also showed that the additive noise contributions $\left(N_{\mathrm{R}}\right.$ and $\left.N_{\mathrm{Q}}\right)$ are negligible compared to the signal dependent noise contribution for sufficiently large intensity values. This simplifies the above equation to

$i_{t}=g_{t}^{\gamma}\left(h_{t} i_{0}+N_{\mathrm{S}}\right)^{\gamma}$.

\section{References}

Altunbasak, Y., Mersereau, R. M., \& Patti, A. J. (2003). A fast parametric motion estimation algorithm with illumination and lens distortion correction. IEEE Transactions on Image Process, 12(4), 395-408.

Cox, I. J., Roy, S., \& Hingorani, S. L. (1995). Dynamic histogram warping of image pairs for constant image brightness. In Proc. IEEE int'l conf. image processing (ICIP) (pp. 2366-2369), 1995.

Greiffenhagen, M., Ramesh, V., \& Niemann, H. (2001). The systematic design and analysis cycle of a vision system: A case study in video surveillance. In Proc. IEEE conf. computer vision and pattern recognition (CVPR) (pp. II:704-711), 2001.

Hager, G. D., \& Belhumeur, P. N. (1998). Efficient region tracking with parametric models of geometry and illumination. IEEE Transactions on Pattern Analysis and Machine Intelligence, 20(10), $1125-1139$.

Harville, M., Gordon, G. G., \& Woodfill, J. (2001). Foreground segmentation using adaptive mixture models in color and depth. In Proc. IEEE workshop detection and recognition of events in video (WDREV) (pp. 3-11), 2001.

He, Y., Wang, H., \& Zhang, B. (2003). Background updating in illumination-variant scenes. In Proc. IEEE int'l conf. intelligent transportation systems (ITSC) (pp. I:515-519), 2003.
Healey, G. E., \& Kondepudy, R. (1994). Radiometric CCD camera calibration and noise estimation. IEEE Transactions on Image Process, 16(3), 267-276.

Hischof, H., Wildenauer, H., \& Leonardis, A. (2004). Illumination insensitive recognition using eigenspaces. Computer Vision Image Understanding, 95(1), 86-104.

Horprasert, T., Harwood, D., \& Davis, L. S. (1999). A statistical approach for real-time robust background subtraction and shadow detection. In Proc. IEEE ICCV'99 FRAME-RATE workshop (pp. II:751-767), 1999.

Hsieh, J.-W., Chang, C.-J., Hu, W.-F., \& Chen, Y.-S. (2003). Shadow elimination for effective moving object detection by Gaussian shadow modeling. Image and Vision Computing, 21(6), 505-516.

Jabri, S., Duric, Z., Wechsler, H., \& Rosenfeld, A. (2000). Detection and location of people in video images using adaptive fusion of color and edge information. In Proc. IEEE int'l conf. pattern recognition (ICPR) (pp. IV:627-630), 2000.

Jacobs, D. W., Belhumeur, P. N., \& Basri, R. (1998). Comparing images under variable illumination. In Proc. IEEE conf. computer vision and pattern recognition (CVPR) (pp. 610-617), 1998.

Javed, O., \& Shah, M. (2002). Tracking and object classification for automated surveillance. In Proc. European conf. computer vision (ECCV) (pp. IV: 343-357), 2002.

Kamikura, K., Watanabe, H., Jozawa, H., Kotera, H., \& Ichinose, S. (1998). Global brightness-variation compensation for video coding. IEEE Transactions Circuits and Systems, 8(8), 988-1000.

McKenna, S. J., Jabri, S., Duric, Z., Rosenfeld, A., \& Wechsler, H. (2000). Tracking groups of people. Computer Vision and Image Understanding, 80(1), 42-56.

Ohta, N. (2001). A statistical approach to background subtraction for surveillance systems. In Proc. IEEE int'l conf. computer vision (ICCV) (pp. II:751-767), 2001.

Pavlidis, I., \& Morellas, V. (2001). Two examples of indoor and outdoor surveillance systems: motivation, design, and testing. In Proc. European workshop advanced video based surveillance systems (AVBS) (pp. 285-296), 2001.

Priebe, C. E. (1994). Adaptive mixtures. Journal of the American Statistical Association, 89(427), 796-806.

Provost, F. J., \& Fawcett, T. (2001). Robust classification for imprecise environments. Machine Learning, 42(3), 203-231.

Scott, M., Niranjan, M., \& Prager, R. W. (1998). Realisable classifiers: Improving operating performance on variable cost problems. In Proc. British machine vision conf. (BMVC) (pp. 306-315), 1998.

Siebert, A. (2001). Retrieval of gamma corrected images. Pattern Recognition Letters, 22(2), 249-256.

Stauffer, C., \& Grimson, W. E. L. (2000). Learning patterns of activity using real-time tracking. IEEE Transactions on Pattern Analysis and Machine Intelligence, 22(8), 747-757.

Toth, D., Aach, T., \& Metzler, V. (2000). Illumination-invariant change detection, In Proc. IEEE southwest symposium image analysis and interpretation (SWSYMP) (pp. 3-7), 2000.

Toyama, K., Krumm, J., Brumitt, B., \& Meyers, B. (1999). Wallflower: Principles and practice of background maintenance. In Proc. IEEE int'l conf. computer vision (ICCV) (pp. 255-261), 1999.

Tsin, Y., Ramesh, V., \& Kanade, T. (2001). Statistical calibration of CCD imaging process. In Proc. IEEE int'l conf. computer vision (ICCV) (pp. I:480-487), 2001.

Wikipedia on Ratio distribution. See http://en.wikipedia.org/wiki/ Ratio_distribution\#cite_note-9.

Withagen, P. J. (2006). Object detection and segmentation for visual surveillance. $\mathrm{Ph} . \mathrm{D}$. thesis, University of Amsterdam.

Withagen, P. J., Schutte, K., \& Groen, F. C. (2004). Probabilistic classification between foreground objects and background. In Proc. IEEE int'l conf. pattern recognition (ICPR) (pp. 31-34), 2004. 
Withagen, P. J., Groen, F. C., \& Schutte, K. (2005). CCD characterization for a range of color cameras. In Proc. IEEE instrumentation and measurement technical conference (IMTC) (pp. III:22322235), 2005.

Withagen, P. J., Groen, F. C., \& Schutte, K. (2007). Ccd color camera characterization for image measurements. IEEE Transactions on Instrumentation and Measurement, 56(1), 199-203.
Withagen, P. J., Groen, F. C., \& Schutte, K. (2008). Shadow detection using a physical basis. In Proc. IEEE instrumentation and measurement technical conference (IMTC) (pp. 119-124), 2008.

Xie, B., Ramesh, V., \& Boult, T. E. (2004). Sudden illumination change detection using order consistency. Image and Vision Computing, 22(2), 117-125. 